\title{
The use of finite element analysis to estimate the changing strength of bone following treatment for osteoporosis
}

\author{
D. B. Burr ${ }^{1,2}$
}

Received: 5 July 2016 / Accepted: 8 July 2016 / Published online: 22 July 2016

(C) International Osteoporosis Foundation and National Osteoporosis Foundation 2016

Finite element analysis (FEA) is a computational technique widely used to estimate the behavior of structures under loading. It is particularly valuable to investigate those conditions which cannot be studied directly by experimentation, and therefore, provides valuable information that cannot be obtained in more direct ways. It has been beneficial in helping to create and optimize non-biological structures for instance, how to engineer an airplane wing to prevent failure from excessive fatigue loading but can also help us to understand how a structure might behave mechanically under various conditions that cannot be reproduced experimentally. Briefly, the technique uses a series of small elements that can be a variety of shapes, and that are connected at nodal points, to computergenerate a morphological structure, such as a hip joint. The elements can be assigned properties that are indicative of the tissue properties that small areas of the biological structure have, either relying on measurements that have been made of the properties of the material, or in those cases in which the properties have not been measured, estimating them based on what we do know. For most models, properties for elastic modulus (tissue stiffness) are assigned to the entire structure, which can be a poor representation of bone tissue properties given one's heterogeneity, rather than to individual elements, A virtual load can be applied to this structure, and the displacement (or deformation) of the structure under that loading condition can be estimated. A second method, known as the

D. B. Burr

dburr@iupui.edu

1 Department of Anatomy and Cell Biology, MS 5035, Indiana University School of Medicine, Indianapolis, IN 46202, USA

2 Department of Biomedical Engineering, Indiana University-Purdue University, Indianapolis, IN 46202, USA flexibility method, is based on known displacements. Knowing the load and the displacement, it is possible to calculate the stresses and strains within the structure, which can provide valuable information to estimate how the structure will "perform" mechanically. Stresses and strains are "derived" parameters that require calculation from the estimated displacements, so are less accurate (or more variable) than the estimated displacements [1]. Nevertheless, the FEA can provide an estimate of the stresses and strains within a structure that cannot be determined in other ways because they cannot be directly measured. If it were possible to do an experiment in which the effect could be directly measured, then FEA is not particularly helpful (just do the experiment). But it is a valuable tool for making predictions about how a structure will behave mechanically and when it might fail in situations in which it is impossible to make such measurements.

Ward et al. [2] have applied this technique in such a case. Long-term treatments with bisphosphonates (BPs) have been suspected of causing bone material to become more brittle, leading to atypical femoral fractures (AFF). This appears to be a time-dependent process. Longer treatment with BPs is associated with deteriorating material properties [3] and a higher risk of sustaining an AFF [4]. Clearly, the properties of human bone cannot be directly measured easily, and especially from people who have been on long-term BP treatment. Yet, the association between BPs and the risk of AFF, and the role that duration of treatment may play, is a vitally important association to understand in the search for preventative measures. The authors sampled 45 iliac crest biopsies from women treated with various BPs (risedronate, alendronate, and ibandronate) singly or sequentially for 1-16 years (mean duration 6.1-8.1 years of treatment, depending on the specific treatment history). Using micro-CT imaging to develop a 3D model of the trabecular structure of the biopsies, they constructed FEA from this patient set from which they could 
estimate apparent stiffness and ultimate stress (stress at failure). In this study, ultimate stress is a global stress calculated by normalizing the failure load to the bone cross-sectional area, rather than a localized ultimate stress in the elements that failed. Adjusting for age and bone volume, they subsequently used polynomial regression analyses and cubic splines to model the data statistically and identify the association between treatment duration and the two mechanical variables. What they discovered is potentially highly significant. The analysis suggested that apparent stiffness and stress at failure, both properties of the cancellous bone structure, will improve over the first 7 years of BP treatment, reaching a maximum at 7.3 years of treatment, and then decrease with longer treatment periods. This prediction coincides exactly with the median duration of BP treatment associated with AFFs $[5,6]$. The clinical importance of this study is that, it suggests that a drug holiday prior to 7 years of treatment might be critically important to prevent the eventual bone fracture. Actually, the study is important more broadly because it does not just suggest that the risk of an AFF may increase with prolonged BP treatment, but in fact, suggests that the fracture of any bone, independent of its mass, may become more likely.

It is important to remember that this is not experimentally verified and probably can never be, but provides a mathematical prediction of what may happen after prolonged treatment. Because it is a mathematical estimate, and not experimentally verified, it is important to understand the limitations that come with it. FEA does not prove that something is true, but only suggests what might happen under certain idealized circumstances, assuming that the circumstances one has entered into the computation are correct (or at least within a reasonable margin of error). The model is only as accurate as the assumptions made in developing the model. As such, FEA might be considered an engine to form hypotheses that can be subsequently tested through experiment or observation, rather than as a conclusive reflection of reality. In this case of course, the hypothesis, that bone structure will deteriorate following the 7 years of BP treatment, is unlikely to be tested in either human or animal bone.

Therefore, it is important that the conditions of the model be verified, that the results be somehow validated, and that the sensitivity of the model to variations in the input parameters be assessed. Although FEA has been used successfully for many years, any new FEA model chooses specific parameters for bone material properties, and determines specific "boundary conditions" under which the model will operate. These will vary for each new FEA model, and; therefore, each new model must be validated.

The validity of the model depends on the choices that are made for the physical and material properties that describe the bone. It is relatively easy to determine the properties of the material that an airplane wing is made of; it is much more difficult to determine the material properties of the bone, not the least because the bone is not homogeneous. Bone's inhomogeneity occurs at several levels of structure. At the ultrastructural level, the mineral content, crystal and collagen maturity, and crosslinking, will vary depending on the mean tissue age, chronological age of the individual, and in some cases, individual's health. Furthermore, unlike an airplane wing, the microstructure of bone is quite variable from person to person. While FEA models can be constructed with gross trabecular architecture that is specific to each individual person, it is much more difficult (or perhaps really impossible) to specify in the model the variations from location to location in the collagen-mineral composite or in microscopic structure, both features which in part determine the tissue properties. As all of these assumptions may reflect reality to different degrees, it is important to validate the model to determine whether it can "predict" a known outcome, perhaps one that has been determined from a separate experiment. The challenge here is that the conditions of the validation experiment may not reflect the conditions of the model precisely.

Bone is anisotropic, meaning that its properties vary depending on the direction of loading. This is particularly difficult to handle in FEA involving cancellous bone as the trabecular struts themselves are running in different directions. Nevertheless, bone is not an isotropic material, and models that assume isotropy are inherently flawed. Moreover, bone is not linearly elastic, but rather viscoelastic. This means that the rate of loading is very important in determining the resulting stress and strain. Bone that is loaded at a higher rate will exhibit stiffer behavior, whereas, bone loaded more slowly will appear to be less stiff.

The sensitivity of the model can provide some assurance that the model is "accurate" or at least a reasonable reflection of reality. In this case, it is important to conduct a parametric analysis, in which the values of various parameters used in the model, for instance, the material properties, can be varied, and the variation in the results based on different values for these properties can be determined. If the model is insensitive to variations in the parameters that are used, then one can have more confidence that the result is plausible. If the model is sensitive to these parameter choices, then any "error" or inaccuracy in choosing those parameters could make the model wildly inaccurate. The sensitivity of the model also depends on the mesh density, i.e., how many elements are used to create the structure. This seems self-evident. Ward et al. [2] state that different mesh densities were explored, and these are apparently published in several Master's theses, although it is not clear if they are easily available.

Other conditions, termed boundary conditions, are also important to the behavior of the model. For instance, it is important for the model to determine how the ends of the bones are constrained during loading. One can imagine that the stresses generated within a bone that is fixed (as when the foot is planted on the ground) will be different than those generated 
when the end of the limb, or bone, is free move (reducing the stresses). Ward et al. [2] have defined these boundary conditions, specifying that the bottom finite element nodes are constrained; whereas the top $5 \%$ of the nodes are subject to $1 \%$ axial compressive strain. However, boundary conditions also will determine how the structure will behave along nonsmooth boundaries, many of which exist in trabecular bone. It becomes a computational challenge to incorporate these conditions, which can affect the predictions made by the model.

One must consider these conditions of validity and sensitivity when deciding whether the results of any FEA model are plausible. Validity and sensitivity depend on which parameters were chosen to describe the properties of the structure, the mesh density, boundary conditions, and conditions of bone's linear elasticity, homogeneity and anisotropy. Ward et al. [2] used an isotropic, homogeneous, and linearly elastic model. A parametric analysis of conditions used in the model would therefore have yielded only proportional differences in apparent modulus and stress. However, as evident from the discussion above, this is not a particularly good reflection of bone's actual viscoelastic or anisotropic properties, or its nonhomogeneity at the tissue level.

One ultimate test of the utility of a model is whether it predicts a plausible result. The results of the Ward study are plausible, though not mechanistic. The model was developed only for trabecular bone properties, and did not incorporate a cortical shell which would carry much of the compressive load. Although not directly applicable to the phenomenon of (AFF), which is a cortical bone failure, it is consistent with the observation that risk of fracture increases with prolonged duration of BP treatment $[4,5]$. The prediction that mechanical properties of bone treated with BPs will decline after 7.3 years, coincides eerily with the median treatment duration associated with AFFs [6, 7]. Therefore, it provides an intriguing confirmation of clinical and experimental observations, but is not directly applicable to the mechanism underlying AFF in cortical bone, both because the model does not incorporate cortical bone, and because it does not consider the changes in material properties that occur with prolonged BP treatment. The model cannot provide a plausible mechanism to explain the predicted decline in bone properties, which in the model are only structural and not material over time. Fragility of the bone with prolonged BP treatment has been proposed to be caused by a change in material properties that make the bone more brittle, and more likely to initiate microdamage that goes unrepaired because of the suppression of remodeling [8]. The FEA model only provides information about changes in bone structure; the model does not vary the material properties over iterations of the model. A continued suppression of remodeling will not cause a decline in BV/TV or any of the architectural parameters associated with cancellous bone strength. Although, it is possible that the model could predict a decline in bone formation at each remodeling site, leading to a loss of bone over time was not measured. There is no real evidence that BPs will, independent of resorption, reduce bone formation at the BMU level. Although, some studies in vitro using high doses of BPs have demonstrated [9-11], and BPs have been shown to suppress mineral apposition rate in rat models [12], no evidence of suppression of net bone formation has ever been shown in either humans or in large animal models. Nor is there evidence of architectural deterioration from prolonged BP treatment in human clinical trials. So, while the results of Ward et al. [2] remain intriguing, particularly in light of what we now know are negative effects of prolonged treatment with BPs on bone, the reason for the effect remains controversial. In that sense, the Ward [2] article is important in pointing out areas in which further experimental work needs to be done, a vital role of the hypothesisformation function that FEA should serve.

Acknowledgments I wish to thank Dr. Joseph Wallace and Dr. Erin McNerny for reading through an earlier draft of the manuscript and making insightful comments which improved the presentation considerably.

\section{Compliance with ethical standards}

Conflicts of interest None.

\section{References}

1. Jacobs CR, Kelly DJ (2011) Cell mechanics: the role of simulation. In: Fernandes PR, Bartolo PJ (eds) Advances on modeling in tissue engineering. Computational methods in applied sciences, vol. 20. Springer, London

2. Ward J, Wood C, Rouch K, Pienkowski D, Malluche HH (2016) Stiffness and strength of bone in osteoporotic patients treated with varying durations of oral bisphosphonates. Osteoporos Int. doi:10.1007/s00198-016-3661-0

3. Burr DB, Liu Z, Allen MR (2015) Duration-dependent effects of clinically relevant oral alendronate doses on cortical bone toughness in beagle dogs. Bone 71:58-62

4. Dell RM, Adams AL, Greene DF, Funahashi TT, Silverman SL, Eisemon EO, Zhou H, Burchette RJ, Ott SM (2012) Incidence of atypical nontraumatic diaphyseal fractures of the femur. J Bone Miner Res 27:2544-2550

5. Burr DB (2016) Bone biomechanics and bone quality: Effects of pharmaceutical agents used to treat osteoporosis. Clin Rev Bone Miner Metab. doi:10.1007/s12018-016-9217-1

6. Shane E, Burr DB, Abrahamsen B, et al. (2014) Atypical subtrochanteric and diaphyseal femoral fractures: second report of a task force of the American Society for bone and mineral research. J Bone Miner Res 29:1-23

7. Shane E, Burr D, Ebeling PR, et al. (2010) Atypical subtrochanteric and diaphyseal femoral fractures: report of a task force of the American Society for bone and mineral research. J Bone Miner Res 25:1-28

8. Ettinger B, Burr DB, Ritchie RO (2013) Proposed pathogenesis for atypical femoral fractures: lessons from materials research. Bone 55:495-500 
9. Idris AI, Rojas J, Greig IR, Van't Hof RJ, Ralson SH (2008) Aminobisphosphonates cause osteoblast apoptosis and inhibit bone nodule formation in vitro. Calcif Tissue Int 82:191-201

10. Orriss IR, Key KL, Colston KW, Arnett TR (2009) Inhibition of osteoblast function in vitro by aminobisphosphonates. J Cell Biochem 106:109-118
11. Koch FP, Yekta SS, Merkel C, Ziebart T, Smeets R (2010) The impact of bisphosphonates on the osteoblast proliferation and collagen gene expression in vitro. Head Face Med 6:12

12. Iwata K, Li J, Follet H, Phipps RJ, Burr DB (2006) Bisphosphonates suppress periosteal osteoblast activity independently of resorption in rat femur and tibia. Bone 39:1053-1058 